\title{
Nicotine derived from the electronic cigarette improves time-based prospective memory in abstinent smokers
}

\author{
Lynne Dawkins ${ }^{1}$, John Turner and Eadaoin Crowe \\ School of Psychology \\ University of East London \\ Water Lane \\ Stratford \\ London E15 4LZ
}

${ }^{1}$ Correponding author: tel.: 02082234421

e-mail: I.e.dawkins@uel.ac.uk

Funding: None

Conflict of Interest:

The first author has a collaborative relationship with Totally Wicked

E-Liquids (TWEL) who provided the electronic cigarettes for this study. TWEL had no involvement in the design, conduct or write up of this study. 


\section{Abstract}

Rationale: It is well-established that nicotine improves, and deprivation impairs, cognitive performance and mood in smokers. Prospective memory (PM), remembering to execute a delayed intention at a given time point, is under-explored in smokers. Whilst a handful of studies have shown improved PM with nicotine, effects of nicotine delivered via the electronic cigarette have not been investigated.

Objective: This study explores whether, by comparison with placebo, nicotine delivered via the e-cigarette can improve PM, tobacco withdrawal symptoms and desire to smoke in abstinent smokers.

Methods: Twenty smokers, abstinent for 8-10 hours, each completed two experimental sessions under nicotine $(18 \mathrm{mg})$ and placebo $(0 \mathrm{mg})$ e-cigarette conditions. Participants completed a single-item desire to smoke scale and the Mood and Physical Symptoms Scale (MPSS). PM was measured using the Cambridge Prospective Memory Test (CAMPROMPT).

Results: Compared with placebo, the nicotine e-cigarette reduced desire to smoke and tobacco withdrawal symptoms, and improved time-based but not event-based PM. There was a moderate, marginally significant negative correlation between PM performance during abstinence and nicotine dependence.

Conclusions: This is the first study to show that nicotine derived via e-cigarette can improve PM in abstinent smokers suggesting efficient nicotine delivery. That the effect of nicotine was restricted to time-based rather than event-based PM is consistent with the view that nicotine acts to improve performance on strategic (effortful) rather than automatic processing. These findings add to the growing body of evidence that the e-cigarette can replace some of the effects of nicotine derived from tobacco smoking thus highlighting its potential for smoking cessation. 


\section{Keywords}

Nicotine, electronic cigarette, prospective memory, event-based, time-based, tobacco withdrawal symptoms, desire to smoke, abstinence

\section{Introduction}

Abstinence from smoking, whether as part of a quit attempt or associated with enforcement of non-smoking laws, is accompanied by tobacco withdrawal symptoms including cigarette craving/desire to smoke, irritability, anxiety, depression and difficulty concentrating (American Psychiatric Association [APA] 2000; Hughes, 2007). Smokers commonly believe that smoking improves attention, alertness and concentration (West, 1993) and such perceived benefits have been suggested to motivate continued smoking (Heishman et al. 2010). A plethora of studies have demonstrated that smoking can improve, and deprivation impair, performance on a variety of cognitive domains including reaction time (Bates et al. 1995; Giannakoulas et al. 2003; Perkins et al. 1995), vigilance/attention (Bell et al. 1999; Gilbert et al. 1997), inhibitory control (Powell et al. 2001), learning (Soar et al. 2008), and working memory (Jacobsen et al. 2005; Merritt et al. 2012). Nicotine replacement therapy (NRT) is also associated with attenuation of tobacco withdrawal symptoms (Kleykamp et al. 2008; Shiffman et al. 2006) and improved cognitive performance in abstinent smokers. Thus sustained attention (Mancuso et al. 1999; Parrot and Craig 1992) and working memory (Atzori et al. 2008; Grobe et al. 1998) are improved after NRT compared with placebo, although there are also some negative findings (Ahnallen et al. 2008; Cook et al. 2003; Warburton and Mancuso 1998).

Nicotine is a cholinergic agonist, binding to presynaptic nicotinic acetylcholine receptors (nAChRs) in turn facilitating the release of a number of neurotransmitters including acetylcholine, dopamine, serotonin and glutamate (Di Matteo et al. 2007). The cholinergic neurotransmitter system is generally considered to play a pivotal role in memory and attention modulation (Kruk-Slomka et al. 2012; Robins and Roberts 2007). Thus nicotinic activation of the prefrontal cortex, parietal cortex, thalamus and hippocampus - areas dense in nicotinic acetylcholine receptors - is thought to mediate its effects on memory and attention (Levin et al. 2006). 
Whilst studies on the effects of abstinence and nicotine administration on working memory abound, prospective memory has received very little attention. Prospective memory (PM) refers to the intention or plan to perform a particular action at some point in the future. This ability to remember to perform a delayed intention is critical to the efficiency of our everyday lives, has been shown to decline with advancing age (Maylor 1996) and is worse in smokers than non-smokers (Heffernan et al. 2005; Heffernan et al. 2010). PM, however, is not a unitary process; it involves encoding and maintaining an intention and retrieving and executing it at the appropriate moment in the future. Neuroimaging studies strongly implicate the rostral prefrontal cortex (Brodmann Area [BA] 10) in PM as well fronto-parietal networks including the inferior parietal lobe (BA 40), precuneus (BA 7), and anterior cingulate (BA 32), regions activated in a wide range of tasks engaging attention, executive functions and working memory (Burgess 2011). Thus PM likely draws on attentional, executive and working memory resources; indeed, previous studies have found a positive relationship between working memory span and PM performance (Smith and Bayen 2005; Smith et al., 2011).

Empirical studies exploring PM functioning have focused on event-based and time-based intentions as both prove to be amenable to laboratory control. Event based PM tasks are characterized by a pre-designated target cue in the environment that when detected, reminds the individual to execute an intention. In contrast, time based intentions must be executed after a specified period of time has elapsed. In both types of PM paradigm, an ongoing distractor task is employed to engage attention and allow the necessary delay required between encoding the intention and executing the action in response to the target cue/elapsed time. Both strategic and automatic processes are thought to be involved in the retrieval of intentions (Einstein and McDaniel 1996; Einstein et al. 2005); the extent to which one or the other is engaged likely depends on the resource demands of the task (Einstein et al. 2005) and presence of external cues to aid retrieval (Nowinski and Dismukes 2005). Time-based PM tasks therefore employ greater strategic processing and self- 
initiated retrieval whilst event-based PM engages more automatic processing given its externally cued nature (Einstein and McDaniel 1990).

Only a handful of studies have explored the effects of acute nicotine administration on PM in deprived smokers and most have used event-based tasks. Rusted et al. (2005) used a lexical decision task with an embedded event-based PM task (in which participants made a response to certain target words or letters). Improved PM performance in $2 \mathrm{hr}$ abstinent smokers was observed after smoking a cigarette. In a later study using the same paradigm, nicotine nasal spray was also shown to improve PM performance in both abstinent smokers and non-smokers (Rusted and Trawley 2006). Using a virtual reality paradigm, the Jansari Assessment of Executive Functions (JEF), our group (Jansari et al. in press) has observed a facilitative effect of $4 \mathrm{mg}$ nicotine gum on event-based and time-based PM in $2 \mathrm{hr}$ abstinent smokers. Whether nicotine is effective in improving PM will likely depend on the speed and efficacy of nicotine delivery to the blood and in turn, engagement with neurotransmitter systems; this may depend on the mode of nicotine administration with more rapid nicotine delivery systems (e.g. smoking, subcutaneous, nasal spray) arguably having a greater effect. Electronic cigarettes are novel nicotine delivery devices which, although unregulated and unlicensed for cessation, are used by smokers as alternatives to smoking, for quitting smoking or to prevent relapse (Etter and Bullen 2011a; Dawkins et al. under review). Their absolute safety and efficacy remain unknown although their potential for smoking cessation/harm reduction is worthy of further exploration.

In order for e-cigarettes to be viewed as an effective smoking cessation/harm reduction method, they should deliver consistent and sufficiently high levels of nicotine to the blood, while simultaneously reducing the desire to smoke and tobacco withdrawal symptoms including the mild cognitive deficits experienced during abstinence. Reports regarding delivery to the blood stream are inconsistent; three human studies reported very low blood nicotine levels in naive users (Bullen et al. 2010; Eissenberg 2010; Vansickel et al. 2010) whilst significant increases in salivary cotinine (a nicotine metabolite; Etter and Bullen 2011b) and blood nicotine levels (Vansickel and Eissenberg 2012) have been shown in regular users. E-cigarettes have been observed to reduce craving/desire to smoke in naive 
users (Bullen et al. 2010; Dawkins et al. 2012; Vansickel et al. 2010) but in some cases, this was also found with the placebo (Omg nicotine) e-cigarette (Dawkins et al. 2012). Likewise, alleviation of withdrawal symptoms did not always differ between placebo and nicotine ecigarette conditions (Bullen et al. 2010; Dawkins et al. 2012). Dawkins et al. (2012) observed a significant reduction in anxiety, poor concentration, irritability and restlessness with nicotine versus placebo e-cigarette but this was only in males. This study was also the first to explore effects of the e-cigarette on cognition by including tasks tapping attention/speed of processing (letter cancellation) and working memory. Whilst attention was not affected, recall on the working memory task was consistently higher in the nicotine group with significant differences emerging with longer periods of interference (i.e. more effortful processing).

We have previously argued (e.g. Dawkins et al. 2007) that cognitive impairments in smokers should be most readily observable during abstinence when background neurotransmitter levels (e.g. dopamine, acetylcholine) are low, compared to after recent nicotine ingestion when neurotransmitter release is stimulated. If the e-cigarette is an effective nicotine delivery tool then we would expect alleviation of abstinence-related negative mood and impaired cognition. The present study therefore aimed to explore whether the nicotine ecigarette improved PM (assessed using the Cambridge Prospective Memory Test, CAMPROMPT; Wilson et al. 2005) tobacco-related withdrawal symptoms and desire to smoke in abstinent smokers using a double-blinded, placebo-controlled, within-subjects design.

\section{Method}

\section{Participants}

Participants were 20 smokers (all Caucasian; 17 employed; 13 female) with a mean age of 31.85 years $(S D=8.7)$, recruited via advertisements, social network forums, e-mail and word of mouth. All smoked within an hour of waking, smoked more than 10 cigarettes a day and had done so for at least one year. 


\section{Design and Data Analysis}

A within-subjects design was employed; each participant was tested on two occasions after overnight abstinence, with e-cigarette type (nicotine vs placebo; order counterbalanced) as the independent variable. The placebo condition therefore represents the 'abstinent' state. Dependent variables were the CAMPROMPT time-based and event-based PM scores, desire to smoke and tobacco withdrawal symptom (MPSS) ratings. Desire to smoke and MPSS variables were analysed using repeated measures analysis of variance with e-cigarette order (nicotine first vs. placebo first) as a between subjects variable. Test order (version A first vs. version B first) was included as an additional between subjects for CAMPROMT PM.

\section{Materials}

\section{The e-cigarette}

The 'Tornado' e-cigarette was supplied by Totally Wicked E-Liquid (TWEL). E-cigarettes were fully charged prior to each assessment session and fitted with either an 18mg (nicotine) or 0mg (placebo) cartridge, both of which were tobacco flavoured.

\section{Breath CO sampling}

Participants were asked to breathe into a Bedfont micro-Smokerlyser for 10 seconds to confirm their abstinence status $(<=10 \mathrm{ppm})$.

\section{Baseline assessment measures}

Demographic information including age, gender, ethnicity and occupation status was collected.

The Fagerström Test of Nicotine Dependence (FTND; Heatherton et al. 1991). A six-item self-report scale to assess nicotine dependence. Scores can range from 0 (low dependence) to 10 (high dependence).

\section{Dependent variables}

Desire to Smoke 
Participants rated their current desire for a cigarette using the single-item 'how strong is your desire to smoke right now?' on a 7-point Likert scale ranging from 'not at all strong' (scored 1) to 'extremely strong' (scored 7).

Mood and Physical Symptoms Scale (MPSS; West and Hajek 2004).

The MPSS was used to measure signs and symptoms commonly associated with tobacco withdrawal. Six items (depressed mood, irritability, restlessness, anxiety, drowsy, difficulty concentrating and hunger) are each rated on a 5-point Likert scale ranging from 'not at all' (scored 0) to 'extremely' (scored 4). A total score in addition to individual items can be computed with scores ranging from 0 to 28 .

The Cambridge Prospective Memory Test (CAMPROMPT; Wilson et al. 2005). The CAMPROMPT comprises six prospective memory tasks which are either cued by events (event-based PM tasks; $\mathrm{N}=3$ ) or by time (time-based PM tasks; $\mathrm{N}=3$ ). There are two parallel versions of the test, $A$ and $B$, which differ only slightly and have been shown to have a high inter-rater reliability, test-retest reliability and parallel form reliability. The inter-rater reliability from the control group showed a correlation between scores of 0.998 (Wilson et al. 2005). Such a high correlation shows that the scoring system devised and described in the CAMPROMPT is both easy to implement and highly reliable. The testretest controls' performances revealed, as expected, there was a practice effect $(z=-3.26$, $p=0.001$ ) however, this was small in magnitude (mean increase $=3.8$ ). For the parallel form reliability there was no significant differences in performance for the group as a whole, for those who did version A first or for those who did version B first, indicating that either version can be given to a participant without the results being significantly affected.

Participants work on a number of 'background' distractor pencil and paper tasks such as a general knowledge quiz or word finder puzzle for 20 minutes. While they are engaged with this, they are asked to remember to do other tasks such as reminding the experimenter of something, either during the 20 minute session or shortly after it finishes. Strategies to aid memory, for example writing a reminder on the paper provided, are permitted. The time interval between being asked to do the task and responding appropriately at the right 
moment are balanced across the cueing conditions. One of the event-based tasks (return a book or keys to the experimenter) is cued by a specific quiz question, one (give a message) by the 'beeper' going off followed by a prompt from the experimenter, and one (find hidden objects) by a statement from the experimenter to say that the session is over. Two of the three time-based tasks (remind experimenter not to forget her keys/mug when there are 7 minutes left until the end of the session; when timer shows 16 mins, change tasks) are cued by a countdown kitchen timer and the third (remind experimenter to telephone reception/garage) is cued by a clock. Both the clock and the timer are on the table in front of the participant. Scores for each subtask can range from 0 to 6 . Six points are awarded if the participant successfully completes the task unaided; four points are awarded if the tasks are completed after the experimenter provides a single general prompt; two points if it is completed after a second more specific prompt and no points if the participant fails to complete the task after two prompts. Total time-based and eventbased CAMPROMPT PM scores were calculated by summing the three time- and eventbased scores for each session, thus scores could range from 0 to 18 with a higher score indicating better PM. Overall total PM was calculated by summing these two.

\section{Procedure}

Participants were asked to remain abstinent overnight (i.e. not to smoke for 8-10 hours) prior to testing on two separate mornings one week apart. Upon arrival at the lab at time 1, participants provided written informed consent and provided a breath sample to confirm compliance with the instructions to remain abstinent from smoking. The experimenter then introduced the participant to the e-cigarette explaining and demonstrating how to use it before allowing the participant to use it ad libitum for ten minutes. Participants were then asked to wait for 15 minutes (to allow time for the nicotine to reach maximum plasma concentration; Bullen et al. 2010; Vansickle and Eissenberg 2012) during which time they completed basic demographic information and the FTND (time 1 only). After 15 minutes, participants then completed the MPSS and desire to smoke scales followed by the CAMPROMPT. 
Testing at time 2 followed the same procedural format using the parallel version of the CAMPROMPT (order counterbalanced), although the demographic questionnaire and the FTND were not repeated. At the end of the second session, participants were debriefed and asked to guess on which occasion they had received nicotine and placebo and then informed accordingly. Each testing session lasted approximately one hour.

The study was granted ethical approval by the University of East London Ethics Committee and was performed in accordance with the ethical standards laid down in the 1964 Declaration of Helsinki.

\section{Results}

\section{Demographics}

Participants were moderately dependent on nicotine as indexed by a mean FTND score of 4.55 (SD: 1.90). Participants complied with the instruction to remain abstinent prior to both testing occasions with a mean CO level of 6.55 (SD: 1.47) prior to the nicotine condition and 6.15 (SD) prior to placebo.

\section{Manipulation Check}

Participants were asked at the end of the second session to guess on which occasion they had received nicotine and placebo. Guessing was at chance level with eight participants guessing correctly, nine guessing incorrectly and 3 reporting that they did not know.

\section{MPSS and craving}

Table 1 displays mean desire to smoke and MPSS scores across the nicotine and placebo ecigarette conditions. All variables were normally distributed. Desire to smoke and overall symptom reporting (total MPSS score) were both significantly higher in the placebo condition $(F(1,18)=11.78, p=0.003 \& F(1,18)=12.17, p=0.003$ respectively). Examination of individual withdrawal symptoms revealed that only anxiety differed 
significantly across conditions with higher anxiety in the placebo condition $(F(1,18)=6.82, p$ $=0.018)$. On no occasion was there a significant interaction with e-cigarette order.

\section{CAMPROMPT}

All CAMPROMPT variables were normally distributed. Overall PM performance was better under nicotine $($ mean $=28.50 ; S D=4.19)$ than placebo $($ mean $=27.40 ; S D=4.37)$. ANOVA revealed that the main effect of e-cigarette type was statistically significant $(F(1,16)=4.44$, $p=0.05)$. Separate analysis for time-based and event-based PM revealed significantly better performance with nicotine for the former $(F(1,16)=5.08, p=0.04)$ but not the latter $(F(1,16)=0.12, p>0.05$; see Figure 1). Event-based PM performance was however, close to ceiling under placebo with six participants scoring the maximum score of 18 and a further 5 scoring 16 . There were no interactions between e-cigarette type and either e-cigarette order or test order for any variable.

Finally, to explore the relationship between nicotine dependence and PM performance, Pearson correlations were performed between FTND and CAMPROMPT scores under both nicotine and placebo conditions. There was a moderate negative correlation between FTND and total time-based PM under placebo which approached significance $(r=-0.41, p=0.07)$. All other correlations were non-significant $(r<0.38, p>0.10)$.

\section{Discussion}

This is the first study to explore effects of nicotine delivered via the e-cigarette on PM. Under double-blinded, placebo controlled conditions, the nicotine e-cigarette improved time-based (but not event-based) PM, desire to smoke and tobacco withdrawal symptoms in abstinent smokers. These findings suggest that that the electronic cigarette can effectively deliver nicotine to impact on cognitive performance although concurrent measurement of blood nicotine levels would be useful to verify this. 
Whilst previous studies have also found positive effects of nicotine on PM, there are some subtle differences. The facilitative effect of nicotine on PM was observed here on timebased but not event-based tasks. This is in contrast to Rusted's group (Rusted et al. 2005, 2009; Rusted and Trawley 2006) who have not included time-based tasks but who have found improvements with nicotine on event-based PM, and with Jansari et al. (in press) who found effects of nicotine gum on both event- and time-based tasks with comparable effect sizes. That differences have emerged between these studies is not surprising given the different methods of nicotine administration and heterogeneous PM tasks employed which vary in the extent to which they engage strategic processing. Nevertheless, whilst the exact nature and mechanism of the nicotine effect requires elucidation, a consistent picture of nicotinic enhancement of PM is beginning to emerge.

Overall performance on event-based compared to time-based PM was generally much better, with performance close to ceiling, and this may have accounted for the lack of improvement by nicotine. Better performance on event-based compared to time-based PM has previously been observed on the CAMPROMPT in smokers, non-smokers (Heffernan et al. 2010), ecstasy/polydrug users or cannabis users (Hadjiefhyvoulou et al. 2011) and is consistent with the multi-process model of PM which proposes relatively greater involvement of either strategic or automatic processing in the retrieval of intentions depending on the resource demands of the task and salience of external cues (Einstein and McDaniel 1990; McDaniel and Einstein, 2000). Thus the greater saliency of the cue in eventbased tasks is proposed to trigger automatic or reflexive retrieval of the intention with active engagement of attention. Time-based PM by contrast, involves greater strategic processing/external monitoring due to the reduced saliency or absence of cues which places greater demand on attentional resources.

The finding that nicotine improved performance on time-based rather than event-based PM in the present study is consistent with this automatic versus strategic dissociation and with the generally accepted view that nicotine, via facilitation of cholinergic transmission, is implicated in the selective enhancement of strategic, effortful-based, working-memory processing rather than more automated processing (Rusted et al. 2005; Sahakian 1998). 
This has been demonstrated using a number of different paradigms, including retrieval induced forgetting (Edginton and Rusted 2003), the antisaccade task (Dawkins et al. 2007), the $n$-back task (Ernst et al. 2001) and most recently by Dawkins et al. (2012) using the Trigram working memory task in which the nicotine e-cigarette improved performance when the demands of the task increased, stretching resources. Nevertheless, this conclusion remains speculative pending replication of this study, utilizing more effortful ongoing tasks in order to reduce the high scores observed on the event-based tasks.

An alternative explanation for the enhancing effects of nicotine on PM based on $\mathrm{FMRI}$ findings, is forwarded by Rusted et al. (2011). It is proposed that nicotine modulates covert attentional reorientation of resources towards PM targets via accelerating deactivation of the default resting brain network (Hahn et al 2007). This is achieved through downregulating activity in parietal regions. This proposal, however, is based purely on the event-based approach to exploring PM thus it is unclear whether this explanation would extend to the effects of nicotine on time-based tasks where cues are absent.

The findings of the present study are interpreted within the context of a 'reversal of abstinence-induced deficits' model, that is prospective memory is impaired during abstinence from smoking and the nicotine e-cigarette is capable of reversing this impairment. It is possible, however, that nicotine may confer a benefit over and above a reversal of deprivation-associated deficits as is suggested by facilitative effects of nicotine in minimally ( $2 \mathrm{hr}$ ) deprived smokers and non-smokers (Rusted and Trawley 2006; Rusted et al. 2009). Ascertaining such a 'true' facilitative effect of nicotine was beyond the scope of this study but it would be interesting to determine whether nicotine derived via the e-cigarette can improve PM in non-smokers and potentially offer another pharmacological intervention to promote cognitive functioning in older adults.

Whilst acute doses of nicotine can improve PM, there is some evidence that chronic smoking can impair it. Heffernan et al. (2010) and Heffernan and O'Neill (2012) have reported impaired PM in current smokers compared with never-smokers and number of years smoked correlated negatively with performance on the CAMPROMPT suggesting that 
a longer smoking history is associated with greater PM impairment. Consistent with this notion, here we observed a moderate but only marginally significant negative correlation between nicotine dependence (as assessed by the FTND) and time-based prospective memory under the placebo (abstinent) but not nicotine condition. Theoretically, this pattern of findings makes sense since it is during abstinence, rather than after acute drug ingestion, that underlying abnormalities of brain functioning are likely to be unmasked (Altmann et al. 1996; Volkow et al. 2004) with greater severity of dependence associated with more severe brain abnormalities (Moreno-López et al. 2012). Nevertheless, given that passive smoking has also recently been associated with impaired self-report PM (Heffernan and O'Neill 2012), is it unclear whether the nicotine per se or some other aspect of tobacco smoking is responsible for the PM deficit.

The present study also aimed to explore effects of the nicotine e e-cigarette on desire to smoke and tobacco withdrawal symptoms and to clarify some of the previous discrepant findings using a better-designed (placebo-controlled, double-blinded, within-subjects) study. The highly significant reduction in desire to smoke, despite the fact that participants were unable to accurately detect on which occasion they had received nicotine, is consistent with previous studies that the nicotine e-cigarette is capable of reducing tobacco craving in abstinent smokers (Bullen et al. 2010; Dawkins et al. 2012; Vansickel et al. 2010). Total MPSS scores were also significantly lower in the nicotine versus the placebo condition consistent with an attenuating effect of the nicotine e-cigarette on tobacco-related withdrawal symptoms. Inspection of individual symptoms however, revealed that only anxiety benefitted from a significant improvement with nicotine. Given that our previous study found greater alleviation of withdrawal symptoms in males, (Dawkins et al. 2012) this may reflect the larger proportion of females in the current study. That there was no effect of nicotine on self-reported ability to concentrate is surprising given the facilitative effect of nicotine on PM. This may reflect the possibility that smokers lack insight into their cognitive state or might be a consequence of the small sample size; with only 20 smokers completing both conditions, there may be insufficient power to detect an effect on individual aspects of tobacco-related withdrawal symptoms. 
To conclude, consistent with a growing body of evidence suggesting that nicotine can improve PM, this study observed a facilitative effect of nicotine delivered via e-cigarette on time-based PM in abstinent smokers. That the improvement was associated with timebased rather than event-based PM is consistent with suggestions that nicotine improves performance when strategic/effortful processing is employed but it could also reflect a ceiling effect observed here. The nicotine e-cigarette also alleviated desire to smoke and tobacco withdrawal symptoms. Taken together, these findings provide further evidence that nicotine delivery via the electronic cigarette is an effective ameliorator of negative mood and mild cognitive impairment associated with tobacco abstention. 


\section{References}

Ahnallen CG, Nestor PG, Shenton ME, MdCarley RW, Niznikiewicz MA (2008) Early nicotine withdrawal and transdermal nicotine effects on neurocognitive performance in schizophrenia. Schizophr Res 100:261-269

Altmann J, Everitt BJ, Glautier S, Markou A, Nutt D, Oretti R, Phillips GD, Robbins TW (1996) The biological, social and clinical bases of drug addiction: commentary and debate. Psychopharmacology 125:285-345

American Psychiatric Association (APA) (2000) The diagnostic and statistical manual of mental disorders. 4th ed. text revision. Washington DC: American Psychiatric Association

Atzori G, Lemmonds CA, Kotler ML, Durcan MJ, Boyle J (2008) Efficacy of a nicotine (4 mg) containing lozenge on the cognitive impairment of nicotine withdrawal. J Clin Psychopharmacol28:667-674

Bates T, Mangan G, Stough C, Corballis P (1995) Smoking, processing speed and attention in a choice reaction time task. Psychopharmacology 120:209-212

Bell SL, Taylor RC, Singleton EG, Henningfield JE, Heishman SJ (1999) Smoking after nicotine deprivation enhances cognitive performance and decreases tobacco craving in drug abusers. Nicotine Tob Res 1:45-52

Bullen C, McRobbie H, Thornley S, Glover M, Lin R, Langesen M (2010) Effect of an electronic nicotine delivery device (e cigarette) on desire to smoke and withdrawal, user preferences and nicotine delivery: randomised cross-over trial. Tob Control 19:98-103

Burgess PW, Gonen-Yaacovi G, Volle E (2011) Functional neuroimaging studies of prospective memory: what have we learnt so far? Neuropsychologia 49:2245-57 
Cook MR, Gerkovich MM, Graham C, Hoffman SJ, Peterson RC (2003) Effects of nicotine patch on performance during the first week of smoking cessation. Nicotine Tob Res 5:169180

Dawkins L, Powell J, West R, Powell J, Pickering (2007) A double-blind placebo-controlled experimental study of nicotine: II - Effects on response inhibition and executive functioning. Psychopharmacology 190:547-467

Dawkins L, Turner J, Hasna S, Soar K (2012) The electronic-cigarette: Effects on desire to smoke, mood and cognition. Addict Behav 37:970-973

Di Matteo V, Pierucci M, Di Giovanni G, Benigno A, Esposito E (2007). The neurobiological bases for the pharmacotherapy of nicotine addiction. Curr Pharm Des 13:1269-84

Edginton T, Rusted JM (2003) Separate and combined effects of scopolamine and nicotine on retrievel-induced forgetting. Psychopharmacology 170:351-357

Einstein GO, McDaniel MA (1990) Normal aging and prospective memory. J Exp Psychol $16: 717-726$

Einstein GO, McDaniel MA (1996) Retrieval processes in prospective memory: Theoretical approaches and some new empirical findings. In M Brandimonte, GO Einstein, MA McDaniel (Eds) Prospective Memory: Theory and Applications (pp 115-142). Mahwah, NJ: Erlbaum

Einstein GO, McDaniel MA, Thomas R, Mayfield S, Shank H, Morrisette N (2005) Multiple processes in prospective memory retrieval: factors determining monitoring versus spontaneous retrieval. J Exp Psychol-Gen 134:327-342

Eissenberg T (2010) Electronic nicotine delivery devices: ineffective nicotine delivery and craving suppression after acute administration. Tob Control 19:87-88 
Ernst M, Matochik JA, Heishman SJ, Van Horn JD, Jons PH, Henningfield JE, London ED (2001) Effects of nicotine on brain activation during performance of a working memory task. Proc Natl Acad Sci USA 98:4728-4733

Etter JF, Bullen C (2011a) Electronic cigarette: users profile, utilization, satisfaction and perceived efficacy. Addiction 106:2017-28.

Etter JF, Bullen C (2011b) Saliva cotinine levels in users of electronic cigarettes. Eur Respir J 38:1219-20

Giannokoulas G, Katramados A, Melas N, Diamantopoulos L, Chimonas E (2003) Acute effects of nicotine withdrawal syndrome in pilots during flight. Aviat Space Environ Med $74: 247-251$

Gilbert DG, Estes SL, Welser R (1997) Does noise stress modulate effects of smoking/nicotine? Mood, vigilance, and EEG responses. Psychopharmacology 129:382-389

Grobe JE, Perkins KA, Goettler-Good J, Wilson A (1998) Importance of environmental distracters in the effects of nicotine on short-term memory. Exp Clin Psychopharmacol $6: 209-216$

Hadjiefhyvoulou F, Fisk JE, Montgomery C, Bridges N (2011) Prospective memory functioning among ecstasy/polydrug users: evidence from the Cambridge Prospectivbe Memory Test (CAMPROMPT). Psychopharmacology 215:761-774

Hahn B, Ross TJ, Yang Y, Kim I, Huestis MA, Stein EA (2007) Nicotine enhances visuospatical attention by deactivating areas of the resting brain default network. J Neurosci 27:3477-89 
Heffernan TM, Ling J, Parrott AC, Buchanan T, Scholey AB, Rodgers J (2005) Self-rated everyday and prospective memory abilities of cigarette smokers and non-smokers: a webbased study. Drug Alcohol Depend 78:235-241

Heffernan TM, O'Neill TS (2012) Everyday prospective memory and executive function deficits associated with exposure to second hand smoke. Addiction 2013: 1-7

Heffernan T, O’Neill T, Moss M (2010) Smoking and everyday prospective memory: A comparison of self-report and objective methodologies. Drug Alcohol Depend 112:234-238

Heatherton TF, Kozlowski LT, Frecker RC, Fagerström KO (1991) The Fagerström Test for Nicotine Dependence: a revision of the Fagerström Tolerance Questionnaire. Br J Addiction 86: $1119-1127$

Heishman SJ, Kleykamp BA, Singleton EG (2010) Meta-analysis of the acute effects of nicotine and smoking on human performance. Psychopharmacology 210:453-469

Hughes JR (2007) Effects of abstinence from tobacco: valid symptoms and time course. Nicotine Tob Res 9:315-27

Jacobsen LK, Krystal JH, Menci WE, Westerveld M, Frost SJ, Pugh KR (2005) Effects of smoking and smoking abstinence on cognition in adolescent tobacco smokers. Biol Psychiatry 57:56-66

Jansari A, Froggart D, Edgington T, Dawkins L (in press) Investigating the impact of nicotine on executive functions using a novel virtual reality assessment. Addiction DOI:

10.1111/add.12082

Kleykamp BA, Jennings JM, Blank MD, Eissenberg T (2008) The influence of transdermal nicotine on tobacco/nicotine abstinence and the effects of a concurrently administered cigarette in women and men. Exp Clin Psychopharmacol 16:99-112 
Kruk-Slomka M, Budzynska B, Biala G. (2012) Involvement of cholinergic receptors in the different stages of memory measured in the modified elevated plus maze test in rats. Pharmacol Rep 64:1066-80

Levin ED, McClernon FJ, Rezvani AH (2006) Nicotinic effects on cognitive function: Behavioral characterization, pharmacological specification, and anatomic localization. Psychopharmacology 184:523-539

Mancuso G, Andres P, Ansseau M, Tirelli E (1999) Effects of nicotine administered via a transdermal delivery system on vigilance: a repeated measure study. Psychopharmacology 142: $18-23$

Maylor EA (1996) Age-related impairment in an event-based prospective memory task. Psychol Aging 11:74-78

Merritt PS, Cobb AR, Cook GI (2012) Sex differences in the cognitive effects of tobacco abstinence: a pilot study. Exp Clin Psychopharmacol 20:258-63

Moreno-López L, Stamatakis EA, Fernández-Serrano MJ, Gómez-Río M, Rodríguez-Fernández A, et al. (2012) Neural Correlates of the Severity of Cocaine, Heroin, Alcohol, MDMA and Cannabis Use in Polysubstance Abusers: A Resting-PET Brain Metabolism Study. PLoS ONE 7(6): e39830. doi:10.1371/journal.pone.0039830

McDaniel MA, Einstein GO (2000) Strategic and automatic processes in prospective memory retrieval: A multiprocess framework. Appl Cognitive Psych 14:S127-S144.

Nowinski JL, Dismukes RK (2005) Effects of ongoing task context and target typicality on prospective memory performance: the importance of associative cueing. Memory 13:649657 
Parrot AC, Craig D (1992) Cigarette smoking and nicotine gum $(0,2$ and $4 \mathrm{mg})$ : effects upon four visual attention tasks. Neuropsychobiol 25:34-43

Perkins KA, Grobe JE, Mitchell SL et al (1995) Acute tolerance to nicotine in smokers: lack of dissipation within 2 hours. Psychopharmacology 118:164-170

Powell J, Dawkins L, Davis R (2001) Smoking, reward responsiveness, and response inhibition: tests of an incentive motivational model. Biol Psychiatry 51:151-163

Robbins TW, Roberts AC (2007) Differential regulation of fronto-executive function by the monoamines and acetylcholine Cereb Cortex 17(suppl 1):i151-i160.

Rusted JM, Ruest T, Gray MA (2011) Acute effects of nicotine administration during prospective memory, an event related fMRI study. Neuropsychologia 49:2362-68

Rusted JM, Sawyer R, Jones C, Trawley SL, Marchant NL (2009) Positive effects of nicotine on cognition: the deployment of attention for prospective memory. Psychopharmacology 202:93-102

Rusted JM, Trawley S (2006) Comparable effects of nicotine in smokers and non-smokers on a prospective memory task. Neuropsychopharmacol 31:1545-1549

Rusted JM, Trawley S, Heath J, Kettle G, Walker H (2005) Nicotine improves memory for delayed intentions. Psychopharmacology 182:355-365

Sahakian BJ (1998) Cholinergic drugs and human cognitive performance. In: Iversen LL, Iversen SD, Snyder SH (Eds) Handbook of psychopharmacology, vol 20. Plenum, London, pp 393-424 
Shiffman S, Ferguson SG, Gwaltney CJ, Balabanis MH, Shadel WG (2006) Reduction of abstinence-induced withdrawal and craving using high-dose nicotine replacement therapy. Psychopharmacology 184:637-44

Smith RE, Bayen UJ (2005) The effects of working memory resource availability on prospective memory: A formal modeling approach. Exp Psychol 52:243-256

Smith RE, Persyn D, Butler P (2011) Prospective memory, personality, and working memory: A formal modelling approach. Z Psychol. 219:108-116

Soar K, Dawkins L, Begum H, Parrott A (2008) The effects of cigarette smoking and abstinence on auditory verbal learning. Hum Psychopharmacol 23:621-7

Vansickel AR, Cobb CO, Weaver MF, Eissenberg TE (2010) A clinical laboratory model for evaluating the acute effects of electronic 'cigarettes': Nicotine delivery profile and cardiovascular and subjective effects. Cancer Epidem Biomar 19:1945-1953

Vansickel AR, Eissenberg T (2012) Electronic cigarettes: effective nicotine delivery after acute administration. Nicotine Tob Res. E-pub ahead of print: Feb 6; doi: $10.1093 / \mathrm{ntr} / \mathrm{ntr} 316$

Volkow ND, Fowler JS, Wang G-J, Swanson JM (2004) Dopamine in drug abuse and addiction: results from imaging studies and treatment implications. Mol Psychiatryn9:557569

Warburton DM, Mancuso G (1998) Evaluation of the information processing and mood effects of a transdermal nicotine patch. Psychopharmacology 135:305-310

West R (1993) Beneficial effects of nicotine: fact or fiction? Addiction 88:589-590 
West R, Hajek P (2004) Evaluation of the mood and physical symptoms scale (MPSS) to assess cigarette withdrawal. Psychopharmacology 177:195-199

Wilson BA, Emslie H, Foley J, Shiel A, Watson P, Hawkins K, et al (2005) The Cambridge Prospective Memory Test. London: Harcourt Assessment 
Table 1: Mean and SD Desire to smoke and MPSS variables under nicotine and placebo e-cigarette conditions

\begin{tabular}{|c|c|c|c|c|}
\hline & \multicolumn{2}{|c|}{ Nicotine } & \multicolumn{2}{|c|}{ Placebo } \\
\hline & Mean & SD & Mean & SD \\
\hline Depressed & 0.45 & 0.69 & 0.45 & 0.69 \\
\hline Irritable & 0.70 & 0.86 & 0.80 & 1.01 \\
\hline Anxious & $0.10^{*}$ & 0.31 & 0.35 & 0.59 \\
\hline Drowsy & 0.20 & 0.52 & 0.30 & 0.57 \\
\hline Restless & 1.05 & 1.00 & 1.15 & 0.88 \\
\hline Hungry & 1.25 & 1.07 & 1.30 & 1.08 \\
\hline Unable to concentrate & 0.65 & 0.81 & 0.70 & 0.80 \\
\hline MPSS Total & $4.35^{* *}$ & 2.92 & 5.00 & 3.03 \\
\hline Desire to smoke & $4.20 * *$ & 1.79 & 4.80 & 1.58 \\
\hline
\end{tabular}


Figure 1: Mean time-based and event-based PM scores under nicotine and placebo conditions (error bars $=1 \mathrm{SE})$

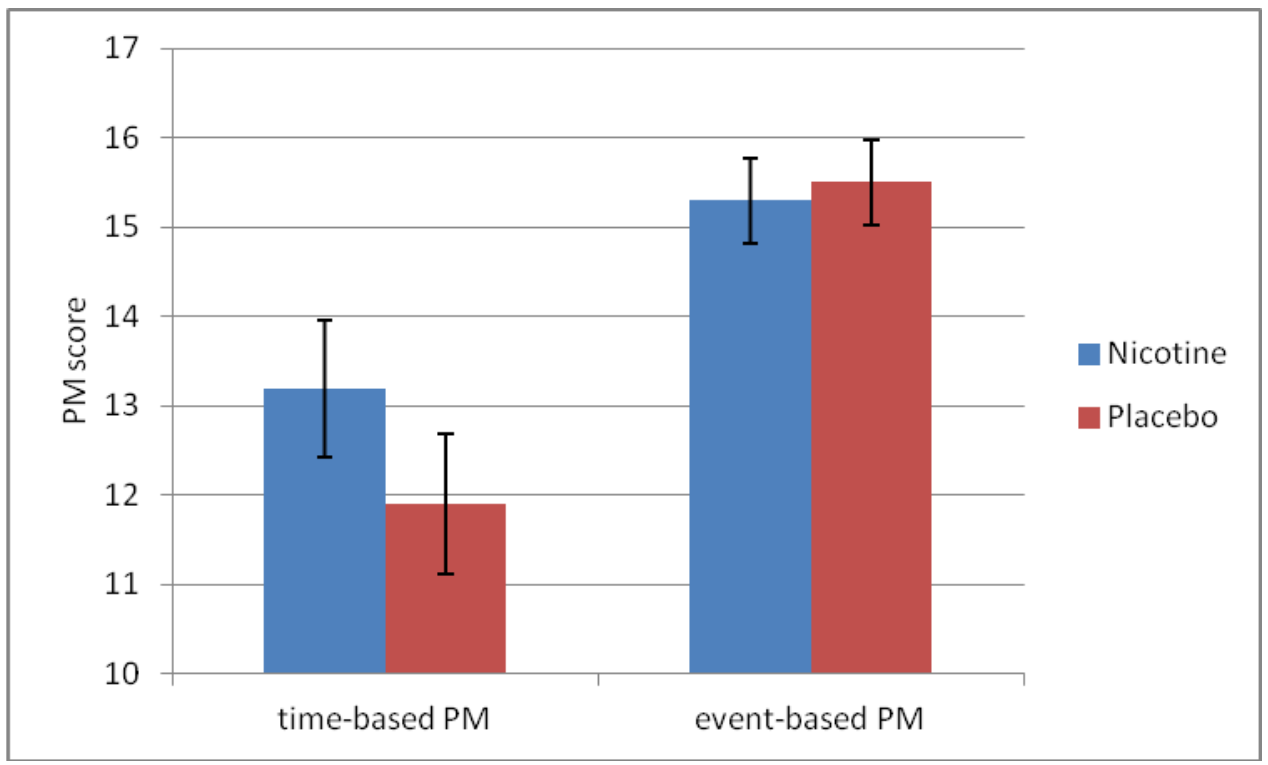

